\title{
An Alternative Treatment for Weakness and Sparseness of Eyebrows: Mesotherapy, A Pilot Study
}

\section{Tahsin Görgülü*}

Department of Plastic, Reconstructive and Aesthetic Surgery, Bulent Ecevit University, Turkey

*Corresponding author: Tahsin Görgülü, Department of Plastic, Reconstructive and Aesthetic Surgery, Bulent Ecevit University, Turkey, Tel: +90-532-351-6064; Fax: +90-372-222-0999; E-mail: tahsinmd@gmail.com

Received date: October 02, 2015; Accepted date: October 10, 2015; Published date: January 01, 2016

Copyright: ( 2016 Gorgulu T. This is an open-access article distributed under the terms of the Creative Commons Attribution License, which permits unrestricted use, distribution, and reproduction in any medium, provided the original author and source are credited.

\begin{abstract}
Introduction: The eyebrow has a significant impact on the perceived beauty of the face, and in today's culture women favour strong and healthy eyebrows. Although eyebrow transplantation and tattooing are two popular techniques for overcoming common concerns about weak and sparse hair growth, both techniques have disadvantages. Mesotherapy was developed in the 1950s, and was initially used to correct hair problems. The proven efficacy of hair mesotherapy has led to the use of the technique for eyebrow problems. Although there have been no published studies of eyebrow mesotherapy in the literature up till today, it is a minimal invasive and efficacious option available for patients.
\end{abstract}

Materials and Methods: A total of 12 patients with complaints of weak and sparse eyebrows (mean patient age: 32 (26-42) years) underwent eyebrow mesotherapy. Of the 12 patients, 8 were satisfied with the results on day 15 after treatment and clinically apparent thickening and darkening of colour was observed.

Discussion: Eyebrow mesotherapy provided good results in this study, which is at odds with the complications of the technique discussed in the literature. In brief, mesotherapy could be useful for correction of eyebrow problems that contribute to the perception of beauty. The results of this pilot study demonstrate that eyebrow mesotherapy is a minimal invasive method that may be tried prior to invasive and irreversible methods, such as eyebrow transplantation and tattooing.

Keywords Weakness; Sparseness; Eyebrows; Mesotherapy

\section{Introduction}

The eyebrow is an important element of facial beauty and weakness and sparseness of eyebrow hair may cause significant psychological problems, especially in women. Eyebrow transplantation and tattooing are popular techniques to correct loss of eyebrow hair. However, transplantation may be complicated by morbidity of the hair transplant, and variation in the hair thickness and growth pattern in comparison to the natural eyebrow, while tattoo can look unnatural.

The field of mesotherapy was founded by Pistor in 1958, and refers to intradermal application of diluted pharmacological agents [1]. As far as we are aware, there have been no previous studies showing the efficacy of mesotherapy, although it has broad applicability and multiple areas of use. However, there have been studies that focus on the complications of mesotherapy [2,3].

Recently, the efficacy of hair mesotherapy has been emphasized in the media but there have been no studies on eyebrow mesotherapy or any emphasis on this application.

In this study, the efficacy of eyebrow mesotherapy was investigated to provide patients with another option other than eyebrow transplantation or tattoo.

\section{Materials and Methods}

A total of 12 patients who complained of weak and sparse eyebrows underwent eyebrow mesotherapy during this pilot study (Figure 1a). Hormone, vitamin, complete blood count (CBC) and other blood test were applied each patient and values were found normal. Therefore, the reason of eyebrows weakness was identified as idiopathic. Patients with abnormal values were excluded from the study and specific treatment applied according to underlying pathology. The mean age of the study participants was $32(26-42)$ years. These patients had declined eyebrow transplantation and tattooing due to their disadvantages, and one session of mesotherapy was planned for each individual. All participants signed patient anonymity and informed consent forms prior to treatment.

Mesotherapy procedures were performed under sterile conditions using a $0.4 \mathrm{~mm}$ length $30 \mathrm{G}$ syringe. In total, $1.6 \mathrm{cc}$ solution was applied to four points in each eyebrow area, $0.2 \mathrm{cc}$ solution at each point (Figure 1b). The drug cocktail included routinely used hair mesotherapy agents. The solution was purchased from Institute BCN ESTHETICS, (Barcelona, Spain) and consisted of $40 \%$ multi-trace elements (zinc sulphate $(0.1 \mathrm{~g})$, copper gluconate $(0.04 \mathrm{~g})$, manganese sulphate $(0.01 \mathrm{~g})$, sodium selenite $(0.002 \mathrm{~g})$, chromium chloride $(0.0004 \mathrm{~g})), 10 \%$ biotin, $20 \% \mathrm{X}-\mathrm{ADN}$ gel (2.5\% deoxyribonucleic acids) and $20 \%$ organic silica + dimethylaminoethanol $(10 \%$ methylsilanol mannuronate, $3 \%$ dimethylaminoethanol). Mesotherapy was applied 3 times with 1 week interval to each patient. 
Citation: Gorgulu T (2015) An Alternative Treatment for Weakness and Sparseness of Eyebrows: Mesotherapy, A Pilot Study. J Cosmo Trichol 1:

Page 2 of 2

\section{Results}

Clinically apparent thickening and darkening of hair colour was observed in eight patients upon assessment at day 15 after treatment (Figure 1c). New hair growth on the edge of the eyebrow was observed in these eight patients, who were satisfied with the results.
Furthermore, positive effect of mesotherapy was still observed in the control at month 2 of the study (Figure 1d). A total of four patients observed no change in their eyebrows after treatment, and comparison of the before and after photos confirmed this result.

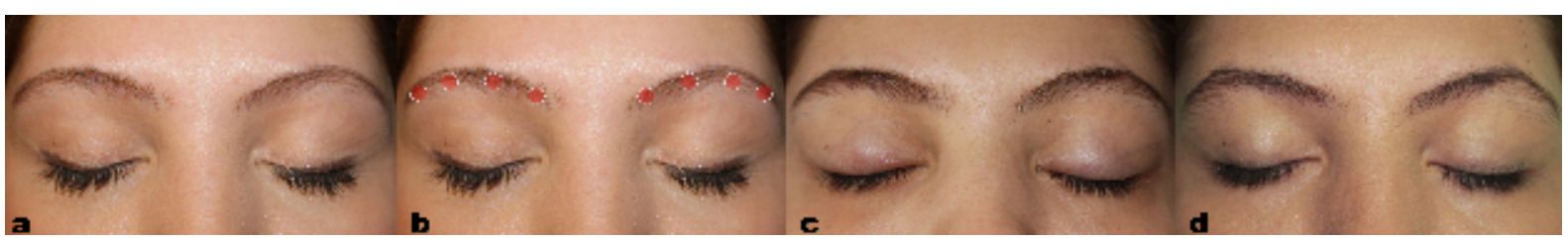

Figure 1: a. Before application, b. Application points, c. Appearance after 2 weeks, d. Appearance in the second month.

\section{Discussion}

Although mesotherapy is not an alternative to surgical treatment, its popularity is gradually increasing due to media attention and patients' desire for minimal invasive procedures. Plastic surgeons typically do not favour these therapies, but also do not emphasize inefficacy of the treatment. The results obtained in this study with a one session of mesotherapy are very encouraging.

The factors leading to weakness of the hair follicles have not been adequately or critically evaluated in the literature [4]. The effects of specific vitamin and mineral deficiencies on hair follicle weakness have been shown [5]. However, there are insufficient data about female-type hair follicle weakness in the literature. Either directly or indirectly, the cocktail used in this study positively affected the eyebrow hair follicle in eight patients. However, no change was observed in four patients, suggesting that these patients may have other causes of hair follicle weakness, which were not addressed by use of our cocktail.

In the light of the results, eyebrow mesotherapy should be considered a minimal invasive method to be tried prior to invasive and irreversible methods, such as eyebrow tattoo and transplantation. Future studies with a larger number of cases and different cocktail components would enable more comprehensive investigation of the advantages of mesotherapy.

\section{References}

1. Herreros FO, Moraes AM, Velho PE (2011) Mesotherapy: a bibliographical review. An Bras Dermatol 86: 96-101.

2. Duque-Estrada B, Vincenzi C, Misciali C, Tosti A (2009) Alopecia secondary to mesotherapy. J Am Acad Dermatol 61: 707-709.

3. Kadry R, Hamadah I, Al-Issa A, Field L, Alrabiah F (2008) Multifocal scalpabscess with subcutaneous fat necrosis and scarring alopecia as a complication of scalp mesotherapy.

4. Mysore v (2010) Mesotherapy in Management of Hairloss - Is it of Any Use? Int J Trichology 2: 45-46.

5. Finner AM (2013) Nutrition and hair: deficiencies and supplements. Dermatol Clin. 31: 167-172. 